\title{
Localization of hand motor activation in Broca's pli de passage moyen
}

\author{
Warren Boling, M.D., André Olivier, M.D., Ph.D., Richard G. Bittar, M.B.B.S., and David \\ Reutens, M.D.
}

Department of Neurosurgery, Montreal Neurological Institute and Hospital, and the McConnell Brain Imaging Center, Montreal, Quebec, Canada

Object. The object of this study was to identify a reliable surface landmark for the hand motor area and to demonstrate that it corresponds to a specific structural component of the precentral gyrus.

Methods. Positron emission tomography (PET) activation studies for hand motor function were reviewed in 12 patients in whom magnetic resonance imaging results were normal. Each patient performed a hand opening and closing task. Using a computer-assisted three-dimensional reconstruction of the surface of each hemisphere studied, the relationship of the hand motor area to cortical surface landmarks was evaluated.

Conclusions. The region of hand motor activation can be reliably identified on the surface of the brain by assessing anatomical relationships to nearby structures. After identification of the central sulcus, the superior and middle frontal gyrus can be seen to arise from the precentral gyrus at a perpendicular angle. A bend or genu in the precentral gyrus is constantly seen between the superior and middle frontal gyrus, which points posteriorly (posteriorly convex). The location of hand motor function, identified using PET activation studies, is within the central sulcus at the apex of this posteriorly pointing genu. The apex of the genu of the precentral gyrus leads to a deep cortical fold connecting the pre- and postcentral gyri and elevating the floor of the central sulcus. This deep fold was described by Paul Broca as the pli de passage fronto-pariétal moyen, and the precentral bank of the pli de passage represents the anatomical substratum of hand motor function. Observers blinded to the results of the activation studies were able to identify the hand motor area reliably after instruction in using these surface landmarks.

Key Words * positron emission tomography * magnetic resonance imaging * motor response * anatomical study * cerebral cortex $*$ hand

The localization of specific motor functions on the precentral gyrus may be difficult to predict despite the well-known top-to-bottom relationship represented by the homunculus.[11,19,23] Activation studies in which positron emission tomography (PET) or functional magnetic resonance (MR) imaging is used can be an aid in identifying the location of sensorimotor function on the precentral gyrus, and this approach has been reliably correlated with cortical stimulation.[16,20,22,30,36] For surgery in the central area, an anatomical landmark by which the practitioner is able to localize a specific motor function can guide cortical stimulation directly to that region, making the stimulation procedure more efficient and safer 
because there are fewer stimulation attempts. In surgery outside the central area, knowledge of functional anatomy is important to guide the boundaries of a resection, and localization of the hand motor area by using gyral and sulcal landmarks may make cortical stimulation and functional neuroimaging unnecessary. In each of these surgical scenarios one can safely consider a smaller craniotomy, shorter operating time, and possibly less expense in preoperative planning.

Hand motor activation on functional neuroimaging occurs in a characteristic omega or knoblike structure that is seen to extend posteriorly from the precentral gyrus on axial MR images.[20,24,29,32,35] This knob has been shown to be a reliable landmark for identification of the hand motor area on axial MR images.[35] However, it is important to identify the structural correlate of the hand motor area on the surface of the brain. With this knowledge, constant anatomical relationships that are present in the central area can be used to identify the hand motor area reliably on visual inspection of the cortical surface and to plan surgery in the region.

In our study, the site of PET motor activation in the hand was evaluated in patients whose MR imaging results were normal in the hemisphere being studied. The purpose of this study was threefold: first, to show that hand motor activation can be reliably identified on inspection of the surface of the brain; second, to demonstrate that hand motor activation is found in a specific structural component of the precentral gyrus, the pli de passage fronto-pariétal moyen (PPFM), a constant anatomical feature of the central area that was originally described by Paul Broca; and third, to determine whether the location of hand motor activation can be reliably predicted in the precentral gyrus by using standard measurements from brain surface landmarks.

\section{CLINICAL MATERIAL AND METHODS}

\section{Patient Population}

We included all PET studies for hand motor activation performed at the Montreal Neurological Institute and Hospital (MNI) in the 3 years prior to 1998 in patients who did not have a lesion in the hemisphere of interest. All the patients had epilepsy, and the PET activation studies were part of a presurgical evaluation. Fourteen hemispheres in 12 patients were included in this study.

There were six males and six females with a mean age of 32 years (range 16-52 years). In two patients both hemispheres were analyzed. On MR images obtained in five patients a lesion was identified contralateral to the hemisphere of interest. The abnormalities consisted of dysplasia, a destructive lesion, atrophy, a previous supplementary motor area resection, and a tumor. Seven of the hemispheres studied contained a nonlesional epileptic focus.

\section{Positron Emission Tomography}

The imaging was performed on a PET scanner (ECAT HR+; CTI/Siemens, Knoxville, TN), with bolus injections of the cerebral blood flow (CBF) tracer $\mathrm{H}_{2}{ }^{15} \mathrm{O}$ (approximately $12 \mathrm{mCi} /$ bolus) administered during baseline and activation states. The scanner produces 63 slices at an intrinsic resolution of $4.2 \mathrm{X}$ $4.2 \mathrm{X} 4 \mathrm{~mm}$. For each patient, at least two baseline scans and two scans for the activation state were performed. This activation task comprised repetitive (approximately $1 \mathrm{~Hz}$ ) hand clenching movements. In the baseline state patients were asked to relax and not move their hands. All scans were performed in a quiet darkened room while the patients' eyes were closed. The PET scans were corrected for attenuation by using a ${ }^{68} \mathrm{Ge}$ transmission scan and reconstructed using a 20 -mm Hanning filter. 


\section{Anatomical MR Imaging}

In all patients, $\mathrm{T}_{1}$-weighted (TR $18 \mathrm{msec}$, TE $10 \mathrm{msec}$, flip angle 30š) MR images were acquired using a three-dimensional (3D) gradient-echo sequence yielding approximately 160 (256 X 256) sagittal images comprising $1-\mathrm{mm}^{3}$ voxels.

\section{Image Analysis}

The MR images were registered into stereotactic space by using a previously described automated algorithm, generating a nine-parameter linear transformation matrix for each image.[5] This

transformation maximized the cross correlation between each patient's MR and target images, consisting of the average of more than 300 normal MR images already registered into stereotactic coordinates.[5,27]

The PET scans were mapped into the same common standard space as the MR images by using an algorithm based on a cross-correlation measure.[10] The PET scans were then normalized for global $\mathrm{CBF}$, and a state-dependent change (change in $\mathrm{CBF}$ ) image volume was obtained. The change in $\mathrm{CBF}$ volume was converted to a t-statistic volume by dividing each voxel by the standard deviation (SD) in change in $\mathrm{CBF}$ pooled across all intracerebral voxels.[9] The statistical significance of t-statistic peaks was determined using a method based on random field theory.[34]

To visualize landmarks on the surface of the brain, a 3D reconstruction of the cortical surface was created by a contouring algorithm that generated a polygonal mesh covering the surface.[14]

\section{Anatomical Study of the Hand Motor Area of the Brain}

A preserved cadaveric brain was stripped of its meninges and surface blood vessels before placing a small pledget soaked with gadopentate (Magnevist; Berlex Canada, Montreal, Canada) was placed over the PPFM. An MR image of the cadaveric brain was obtained (flip angle 30š, TR $22 \mathrm{msec}$, TE $10 \mathrm{msec}$, 200-mm field of view, 150 1-mm-thick slices. Additionally, a 3D surface reconstruction of the MR images was generated as previously described.[14] A preserved cadaveric brain stripped of meninges and surface blood vessels was also used for dissection of the central area at the level of the PPFM .

\section{Brain Measurements}

We evaluated the distance from brain surface landmarks on or near the precentral gyrus to the peak of hand motor PET activation in each hemisphere. The brain surface landmarks were the interhemispheric fissure, the superior frontal gyrus (F1), the middle frontal gyrus (F2), and the sylvian fissure. The F1 and F2 landmarks were the midpoint of their gyral crowns at their origin from the precentral gyrus. The measurements were made both along a straight line and following the gyral bends within stereotactic coordinate space. The euclidean distance between two points was calculated using the formula

$$
\sqrt{ }\left(x 1-x_{2}\right) z+\left(y_{1}-y_{2}\right) z+\left(z_{1}-z_{2}\right) z
$$

Measurements following the curves of the precentral gyrus were performed on the computer workstation by tracing a line on the 3D surface rendering. Additionally, stereotactic coordinates were obtained for the peak of each PET activation on the 3D surface rendering.

\section{Interobserver Reliability}

The ability of trained observers to predict the site of hand motor activation by using cortical surface 
anatomical landmarks was examined. Two methods of identifying the hand motor area were compared. The first was that of Yousry, et al.,[35] which relied on the identification of a "knoblike structure" on the axial MR image through the precentral gyrus. The second method, described herein, entailed the identification of cortical landmarks by using a 3D brain surface reconstruction. The instruction provided to observers in this study is described in detail in the Appendix. In brief, the observer was asked to identify the posterior pointing bend in the precentral gyrus found between the superior and middle frontal gyri. The site of hand motor activation is located in the apex of this posterior pointing bend.

The two methods of hand motor identification were described to the observers just before the testing by using a training set containing the article of Yousry, et al.,[35] and the Appendix. Five physicians blinded to the PET activation studies used each method to identify the site of hand motor activation on the MR image and on the surface of the 3D brain reconstruction of the 12 patients' images. The observers' response was considered correct if it fell within $1 \mathrm{~cm}$ of the peak voxel of the PET activation focus and within the correct gyrus. Comparison of correct responses with each method was performed using the chi-square analysis.

\section{RESULTS}

\section{Functional Anatomy of the Hand Motor Area of the Brain}

In all the hemispheres studied, the area of hand motor activation was found to be located on the posterior bank of the precentral gyrus or within the central sulcus in the hemisphere contralateral to the motor task. Each area of activation also was seen to be located between the intersection of the superior and middle frontal gyri with the precentral gyrus (Fig. 1).

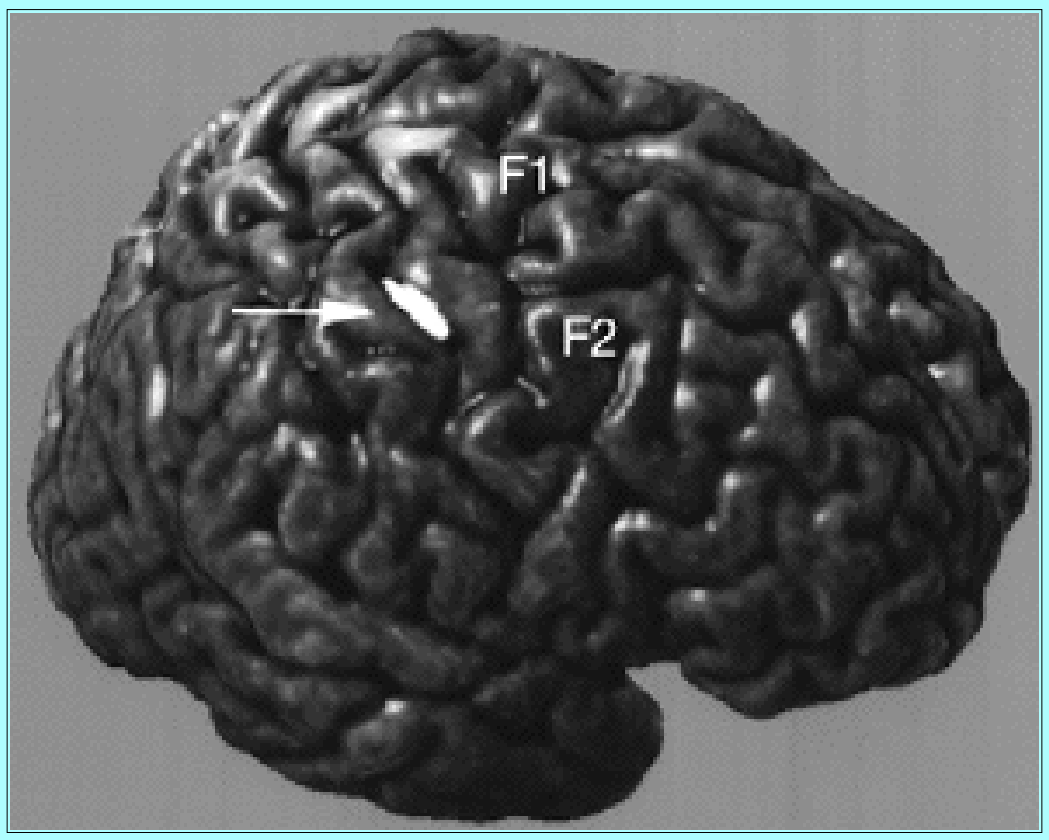

Fig. 1. A 3D reconstruction of cortical surface coregistered with PET activation. Arrow points to hand motor activation in Broca's middle bend, seen as a white area on the posterior bank of the precentral gyrus.

At this level, in each of the patients studied, a bend was found in the precentral gyrus that pointed posteriorly and corresponded to Broca's middle bend of the central sulcus; we used this nomenclature.[2] The hand motor activation was found to occur at the apex of the middle bend in all patients in our study. 
The finding of the hand motor area within the knob- or omega-shaped area of the precentral gyrus has already been described,[20,24,29,32,35] and the axial MR image obtained during the hand motor activation in each of our patients confirmed this observation (Fig. 2).

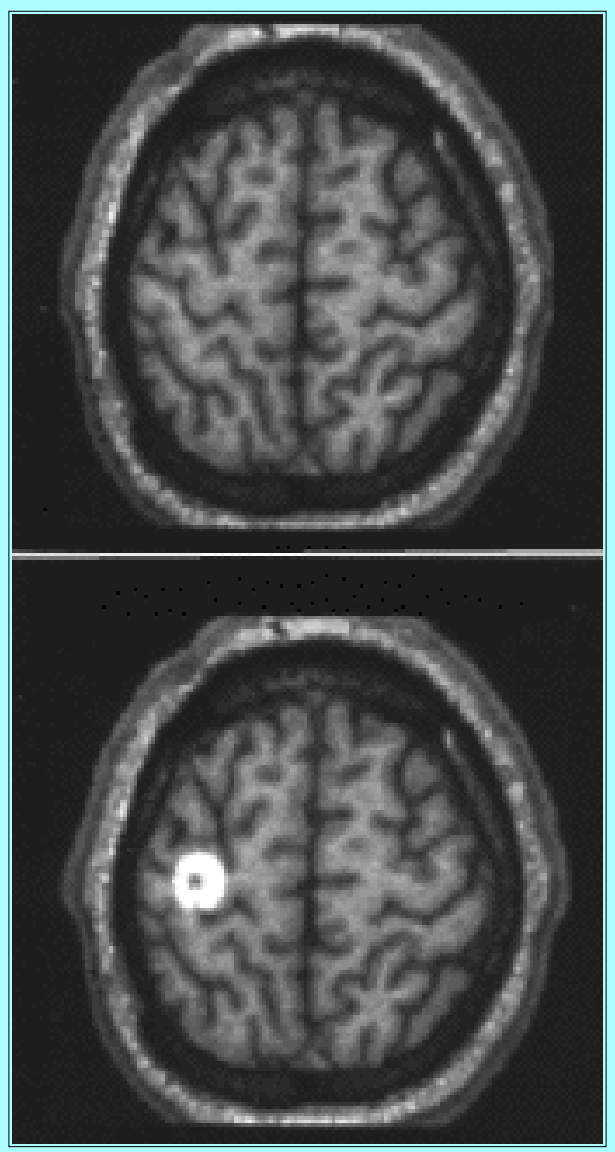

Fig. 2. Upper: Axial MR image without PET activation demonstrating the knob in the precentral gyrus that is the location of hand motor activation. Lower: The same axial MR image through the hand area coregistered with hand motor PET activation.

The constancy of this landmark predicted a fundamental anatomical relationship to the hand motor area, and several key features were noted when the cadaveric brain was examined. Broca's middle bend was found between the superior and middle frontal gyri. When the pre- and postcentral gyri were pried apart at the middle bend, a deep bridge of cortex was seen connecting the two, giving rise to a distinct bulge from the posterior wall of the precentral gyrus and elevating the floor of the central sulcus (Fig. 3). 


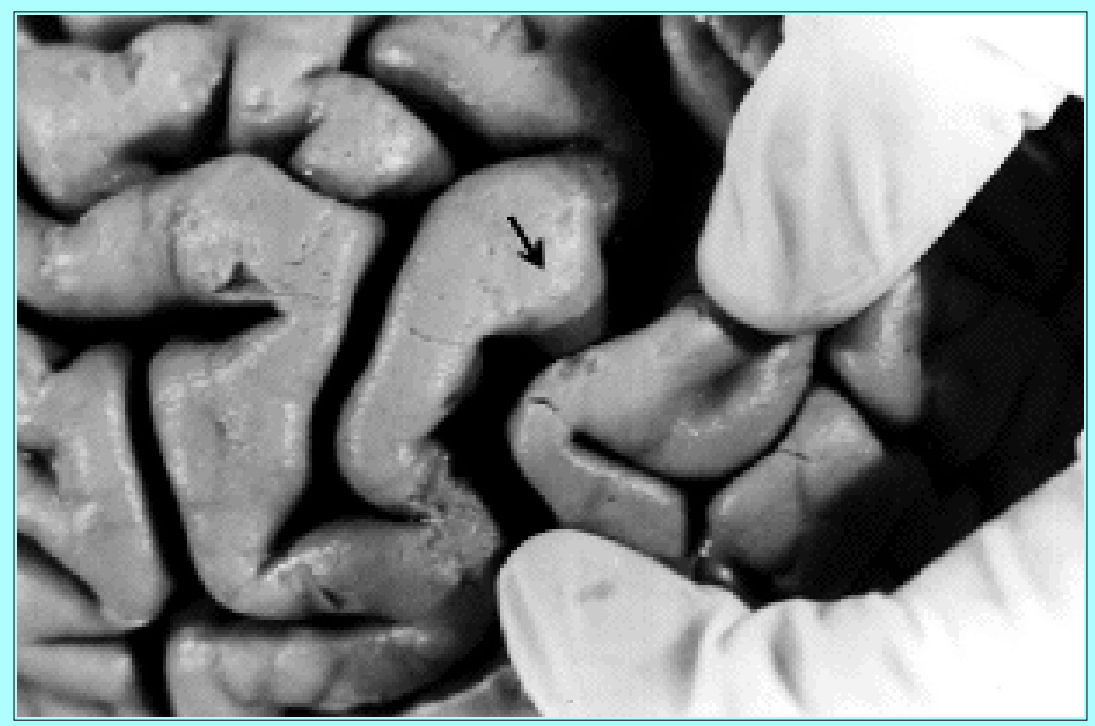

Fig. 3. Photograph of cadaveric brain with the postcentral gyrus retracted to expose the central sulcus. Arrow points to the bulge from the precentral gyrus into the central sulcus at the level of the middle bend.

This constitutes Broca's PPFM. To further delineate the anatomical correlate of hand motor activation, a pledget soaked in gadopentate was placed over the PPFM on the posterior wall of the precentral gyrus in the cadaveric brain. The MR image and subsequent 3D surface reconstruction of the cadaveric brain demonstrated that the location of the pledget corresponded convincingly with the location of hand motor activation in the patients in the study (Fig. 4 left). The MR image also showed that the pledget lay over the knob or omega of hand motor function (Fig. 4 right). When the postcentral gyrus of the cadaveric brain was removed, the anatomical features of the PPFM could be seen more clearly (Fig. 5).
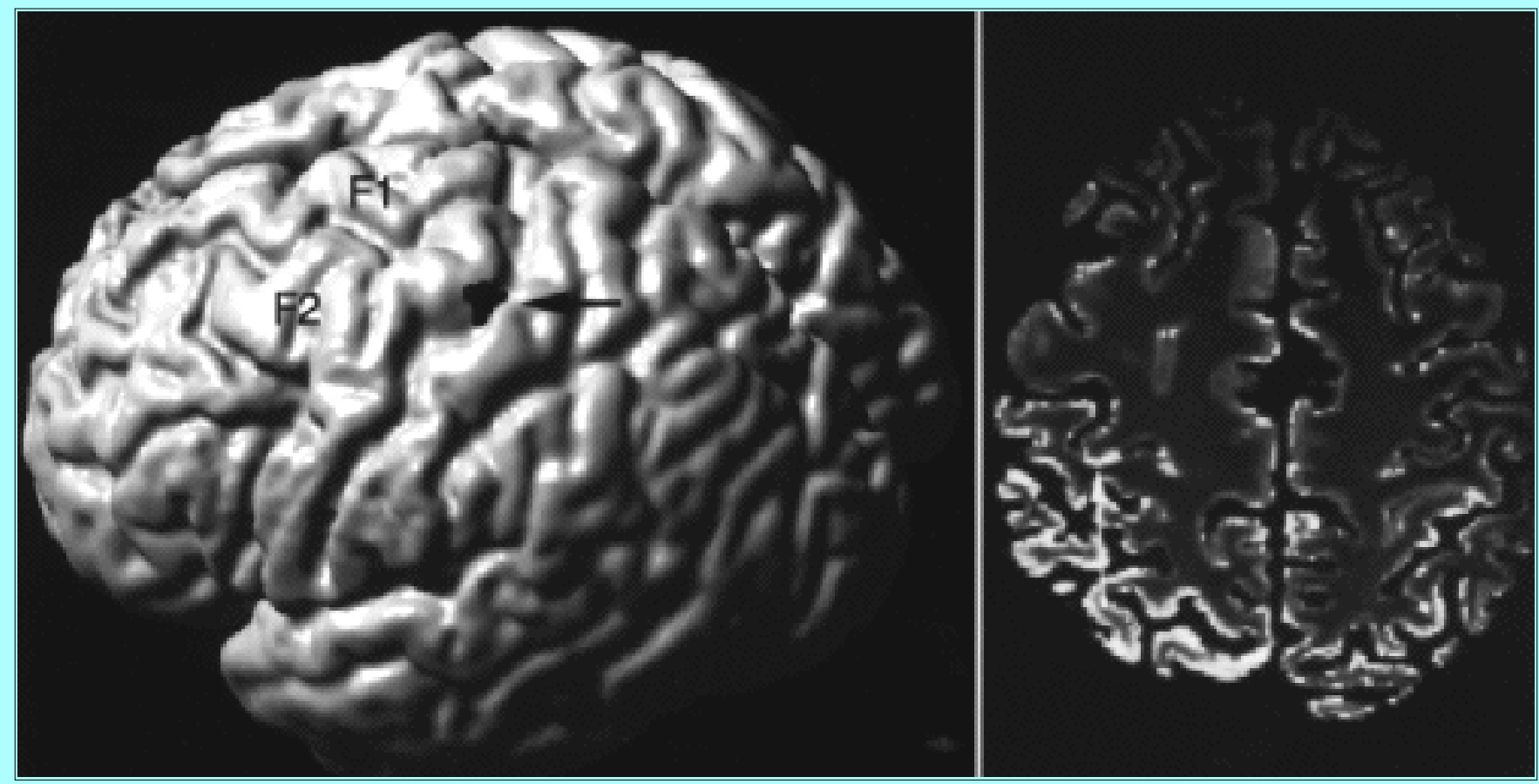

Fig. 4. Left: A 3D reconstruction of an MR image obtained in a cadaveric brain. Arrow points to the location of a gadopentate-soaked pledget (reconstructed as a black object) placed over the PPFM. Right: Axial MR image of cadaveric brain. Arrow demonstrates gadopentate-soaked pledget over the PPFM. This corresponds with the slice from the axial MR image obtained in a living patient and shown in Fig. 2. 


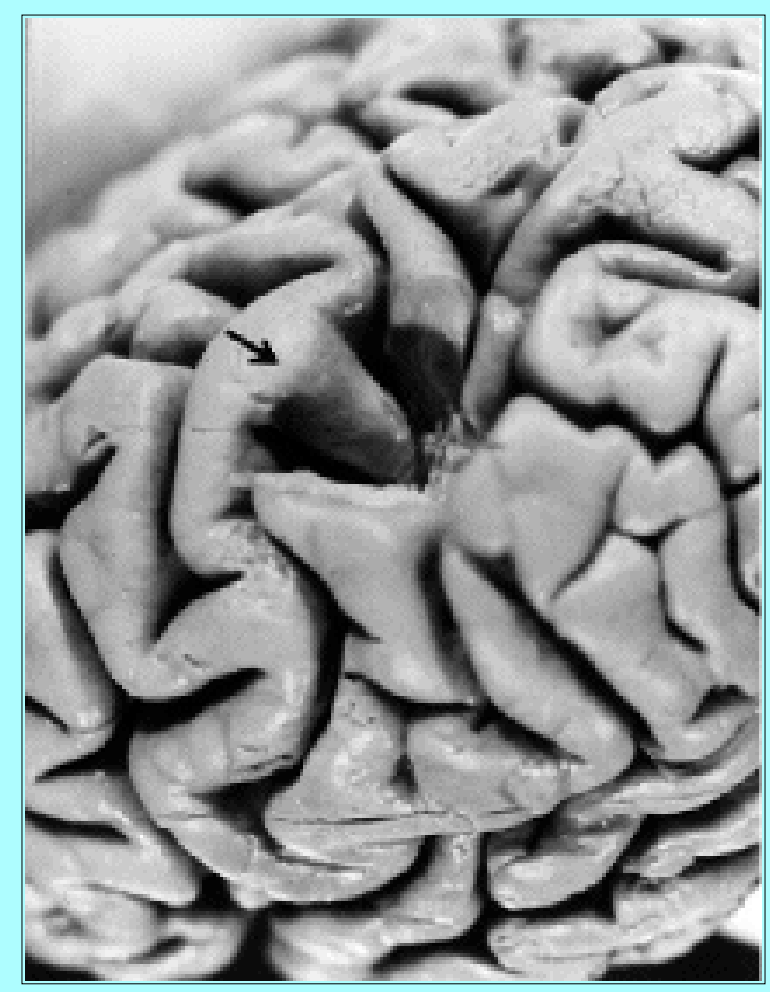

Fig. 5. Photograph of cadaveric brain in which the postcentral gyrus has been removed at the level of Broca's middle bend. Arrow points to the PPFM bulging from the posterior wall of the precentral gyrus. This is the anatomical substratum of the hand area.

\section{Interobserver Reliability}

Observations by physicians blinded to the sites of activation yielded a $92 \%$ accuracy ( 55 of 60 ) in correctly predicting hand motor activation on the surface of the brain, and $88 \%$ accuracy (53 of 60) in correctly predicting hand motor activation by using the technique of knob identification on axial MR images. There was no significant difference between the two methods in the observers' ability to predict hand motor localization $(\mathrm{p}>0.1)$.

\section{Brain Measurements}

The length of a straight line between the hand motor activation and a surface landmark revealed a range of measurements (Table 1). The activation to sylvian fissure measurements had the least coefficient of variation $(9 \%)$ and the activation to $\mathrm{F} 1$ measurements the greatest $(25 \%)$. The mean distance from the sylvian fissure to the activation was $61 \pm 5.4 \mathrm{~mm}$ standard deviation (SD). 


\begin{tabular}{|c|c|c|c|c|}
\hline \multicolumn{5}{|c|}{$\begin{array}{c}\text { TABLE } 1 \\
\text { DISTANCE FROM A SURFACE LANDMARK TO THE PEAK OF HAND MOTOR ACTMATION } \\
\text { MEASURED IN A STRAIGHT LINE ON } 14 \text { PET SCANS } \\
\text { OBTANED IN } 12 \text { PATIENTS WITH EPILEPSY }\end{array}$} \\
\hline \multirow[b]{2}{*}{ PET Study } & \multicolumn{4}{|c|}{ Distance to Activation (mm) } \\
\hline & F1 & Sylvian & Fissure & Midine \\
\hline $\begin{array}{c}1 \\
2 \\
3 \\
4 \\
5 \\
6 \\
7 \\
8 \\
9 \\
10 \\
11 \\
12 \\
13 \\
14 \\
\text { median } \\
\text { mean } \pm \text { SD } \\
\text { coefficient of wariation (\%) }\end{array}$ & $\begin{array}{l}36 \\
42 \\
34 \\
48 \\
15 \\
43 \\
38 \\
25 \\
35 \\
32 \\
32 \\
27 \\
28 \\
31 \\
33 \\
33 \pm 8.2 \\
25\end{array}$ & $\begin{array}{c}28 \\
36 \\
18 \\
27 \\
31 \\
26 \\
15 \\
28 \\
16 \\
27 \\
25 \\
25 \\
23 \\
33 \\
21 \\
25 \pm 5.9 \\
24\end{array}$ & $\begin{array}{l}60 \\
65 \\
67 \\
47 \\
69 \\
56 \\
58 \\
63 \\
57 \\
61 \\
63 \\
63 \\
66 \\
62 \\
19 \\
61+5.4 \\
9\end{array}$ & $\begin{array}{l}43 \\
49 \\
45 \\
59 \\
35 \\
49 \\
50 \\
42 \\
43 \\
40 \\
43 \\
35 \\
38 \\
41 \\
24 \\
44.6 .6 \\
15\end{array}$ \\
\hline $\begin{array}{l}\text { "Measurements from th } \\
\text { (P2) begin at the mid point }\end{array}$ & m nor & $\begin{array}{l}\text { Us }(F) \\
\text { tersed }\end{array}$ & mia & ital g! \\
\hline
\end{tabular}

Measurements that followed the curves of the precentral gyrus from the hand motor activation to surface landmarks showed the coefficient of variation to fluctuate from 11 to $32 \%$ (Table 2). The length of the measurement from the sylvian fissure to the hand activation showed the least coefficient of variation (11\%), with a mean of $96 \pm 11 \mathrm{~mm}$ (SD). Additionally, the greatest variation was the length between F1 and hand activation $(32 \%)$. 
TABLE 2

DISTANCE TO ACTMATION MEASURED ALONG THE CROWN AND FOLLOWING THE CURVES OF THE PRECENTRAL GVRUS IN 3D SPACE*

\begin{tabular}{|c|c|c|c|c|}
\hline \multirow[b]{2}{*}{ PET Study } & \multicolumn{4}{|c|}{ Distance to Activation (mm) } \\
\hline & F 1 & $\mathrm{~F} 2$ & $\begin{array}{l}\text { Sylvian } \\
\text { Fissure }\end{array}$ & Midine \\
\hline 1 & 40 & 27 & 89 & 63 \\
\hline 2 & 59 & 39 & 87 & 69 \\
\hline 3 & 30 & 24 & 105 & 55 \\
\hline 4 & 57 & 18 & 81 & 59 \\
\hline 5 & 39 & 49 & 104 & 42 \\
\hline 6 & 48 & 34 & 88 & 56 \\
\hline 7 & 66 & 14 & 81 & 64 \\
\hline 8 & 22 & 44 & 108 & 44 \\
\hline 9 & 35 & 32 & 90 & 62 \\
\hline 10 & 39 & 31 & 118 & $4 \overline{5}$ \\
\hline 11 & 30 & 43 & 101 & 43 \\
\hline 12 & 32 & 33 & 97 & 35 \\
\hline 13 & 35 & 42 & 104 & 42 \\
\hline 14 & 28 & 27 & 91 & 52 \\
\hline median & 44 & 35 & 37 & 34 \\
\hline mean $\pm \mathrm{SD}$ & $40 \pm 12.9$ & $33 \pm 10.2$ & $96 \pm 11$ & $52 \pm 10$ \\
\hline coefficient of wariation (\%) & 32 & 31 & 11 & 19 \\
\hline
\end{tabular}

The $\mathrm{X}, \mathrm{Y}$, and $\mathrm{Z}$ stereotactic coordinates obtained for each point of hand activation revealed coefficients of variation from 5 to $15 \%$ for right hemisphere activations and 9 to $17 \%$ for left hemisphere activations (Table 3 ). The $\mathrm{Z}$ coordinate (superoinferior) showed the least variation for right and left side activation. 


\begin{tabular}{|lccc|}
\hline \multicolumn{4}{c}{ TABLE 3 } \\
\multicolumn{4}{|c|}{ STEREOTACTK COORDINATS MEASURED AT THE PEAK OF } \\
HAND MOTOR ACTMATION
\end{tabular}

\section{DISCUSSION}

Broca's observations on aphasia popularized the notion that specific cortical functions can be localized on the surface of the brain.[25] This idea gave a new importance to the surface convolutions, and Broca was one of the early scientists to make meaningful and careful descriptions of cortical anatomy. His observations concerning the precentral gyrus led him to describe three folds connecting the pre- and post central gyri: the pli de passage fronto-pariétal supérieur found at the interhemispheric fissure (paracentral lobule), the pli de passage fronto-pariétal inférieur separating the central sulcus from the sylvian fissure (subcentral gyrus), and the PPFM. The PPFM is described by Broca as a bulge into the central sulcus below the level of the superior genu. It is always located within the sulcus, requiring the gyri to be pulled apart to visualize it, but it elevates the floor of the central sulcus and connects the preand postcentral gyrus (Figs. 3 and 5). Broca saw it rise to the height of the gyral crown and divide the sulcus only once, but, he said, this was an anomaly.[2]

\section{Anatomy of the Central Area}

The central sulcus always takes a sinusoidal shape, but the number of bends or genua described varies depending on the author. Cunningham[6] suggested that there are two bends in the rolandic fissure: "The superior genu is weaker and is always directed backwards ... The inferior genu is always stronger and looks forwards." Campbell[3] also attributed two genua to the rolandic fissure: "The superior genu is a backward curve ... the inferior genu is not as constant and is a very unstable element." Testut and Latarjet[28] described three genua of the central sulcus. The superior and inferior genua were convex anteriorly, and the middle genu was convex posteriorly. Both Broca[2] and Dejerine[8] had already 
described a superior and an inferior genu of the central sulcus, both convex anteriorly, and a middle bend convex posteriorly. Dejerine indicated that the middle bend is near the insertion of F2. The superior genu of Cunningham and Campbell, as well as the middle genu of Testut and Latarjet, corresponds to Broca and Dejerine's middle bend. Broca's middle bend, which lies between the middle and superior frontal gyrus with its concavity directed anteriorly, was a constant feature in each of our patients (Fig. 1).

Broca's PPFM has been described by more contemporary anatomists and given other names. Cunningham,[6] in 1892, described, in the region of his superior genu, two deep interdigitating gyri from the pre- and postcentral convolutions. He went on to say: "They unite at the bottom of the sulcus in the form of a distinct deep gyrus." Campbell,[3] in 1905, also described the anatomy near his superior genu of the fissure of Rolando, and noted "that when the lips of the fissure of Rolando are drawn apart, a deep annectant gyrus, giving rise to some shallowing of the fissure, is displayed in the vicinity of the upper genu." He went on to describe buttresses springing from the apposed walls of the fissure and in his opinion, "the most important element in the formation, is the large bulging buttress on the precentral wall, for the accommodation of which the post-central wall suffers indentation." Campbell also made the important observations that the precentral buttress is always placed "at the level of the superior genu" (Broca's middle bend), and at the "same horizontal level as the superior frontal sulcus." Both Cunningham and Campbell referred to the deep gyrus connecting the pre- and postcentral convolutions as an "annectant gyrus."

More recent investigators have also described some of the features seen in the middle bend region of the central sulcus. White, et al.,[32] described a prominent interdigitation of the walls of the pre- and postcentral gyri, which they recognized in the depths of the central sulcus. They also noted that published figures demonstrating functional neuroimaging with hand motor activation have invariably shown the activation to lie in this region. Wood, et al.,[33] noted that hand function may occur at a bend in the central area, but it was not a consistent observation. Suk, et al.,[26] used 3D surface rendering of the central area to demonstrate hand somatosensory activity with magnetoencephalography. The 3D reconstruction they obtained shows a posterior bulge from the precentral gyrus that corresponds to the structure herein described as Broca's PPFM. Examination of the figures from Puce and colleagues[21,22] reveals hand motor activation in the precentral gyrus at the level of the middle bend on 3D brain surface reconstructions. In several modern studies of cortical stimulation a close analysis of the illustrations shows hand motor function in the precentral gyrus at the middle bend.[12,21,36]

The finding of hand motor activation within the precentral gyrus at the middle bend is consistent with cortical stimulation studies.[7,18,19] Cortical stimulation, however, clearly yields hand and finger movements over a larger area than encompassed by the PPFM. Stimulation can produce individual finger movements that extend well inferior to the middle bend, with thumb movement being the most inferiorly located.[7,18,19] The stimulation studies of Penfield and colleagues[18,19] show hand and finger movements elicited over half or more of the precentral gyrus on the lateral convexity. The motor task used in the present study, hand opening and closing, involves movements of the fingers and hand. The center of PET activation in the precentral gyrus corresponds to the region of peak change in $\mathrm{CBF}$ resulting from the dominant motor activity. The PET activation resulting from thumb movement is probably underrepresented in the present series because the task paradigm favors far greater finger than thumb movement. Also, the signal-to-noise ratio is likely to be too low for significant activation peaks of individual finger movements. Catalan, et al.,[4] showed finger somatotopy in the primary somatosensory cortex by using PET activation and by repeating each condition five times to improve the signal-to-noise ratio of individual fingers. Other authors have also shown finger somatotopy of the somatosensory cortex 
in functional MR imaging studies.[13,15]

\section{Primary Motor Cortex}

In each patient studied here the peak of PET activation occurred within the central sulcus on the posterior bank of the precentral gyrus. Cortical stimulation will invariably show responses over the crown of the precentral gyrus.[18] However, the actual pyramidal cell activity must occur within the sulcus. The cytoarchitectonic area of the cerebrum that contains agranular isocortex with giant pyramidal (Betz) cells is the primary motor cortex.[31] In the brain maps published by Bailey and von Bonin,[1] Brodmann named this Area 4; for von Economo it was FA-gamma; and Campbell called this the precentral or motor area. The brain maps of each of these authors show a general retreat of the anterior border of the motor area into the central sulcus as one progresses inferiorly along the precentral gyrus from the midline to the sylvian fissure.[31] Campbell[3] noted that in the lower third of the precentral gyrus, the cytoarchitectonic field comprising Betz cells was hidden within the central sulcus and confined to the posterior wall of the precentral gyrus. White, et al.,[32] found the anterior border of area 4 to be at the lip of the anterior bank of the central sulcus at the level of the junction of the superior frontal and precentral gyrus. More inferiorly along the precentral gyrus, area 4 recedes deeper into the central sulcus. These authors also noted that area 4 enlarged its surface area at the level of Campbell and Cunningham's annectant gyrus, which is Broca's PPFM.

\section{Measurements to Hand Area}

Penfield and colleagues[18,19] concluded that a specific motor function could not be reliably identified by any measurement along the precentral gyrus. A wide range of distances was found here when hand motor activation was measured from different points of reference in or near the precentral gyrus. The straight line measurement from the sylvian fissure to the hand motor activation produced the smallest range $(19 \pm 5.4 \mathrm{~mm}, \mathrm{SD})$ and coefficient of variation $(9 \%)$. Although this would be the most reliable measurement, a range of $19 \mathrm{~mm}$ spanned $20 \%$ of the average distance from the midline to the sylvian fissure along the precentral gyrus in our patients. These findings may still have a useful application: in surgery of the central area a resection of less than $47 \mathrm{~mm}$ from the sylvian fissure would preserve the hand motor area in the patients studied here. This does not take into account the thumb area, which is more inferiorly placed. Additionally, the mean distance from the sylvian fissure to the hand area (61 \pm $5.4 \mathrm{~mm}, \mathrm{SD})$ can be used to estimate the hand motor area localization if the middle bend is not identified. The use of stereotactic coordinates to identify hand motor activation demonstrated the greatest range of variability between patients in the $\mathrm{X}$ (mediolateral) direction, which is primarily along the long axis of the precentral gyrus, and the most important plane for hand motor localization after the precentral gyrus has been identified. The least range for a stereotactic coordinate was in the $\mathrm{Y}$ (anteroposterior) and Z (ventrodorsal) directions, which are more useful for localizing the precentral gyrus. The relatively constant location of the precentral gyrus within a stereotactic space has been demonstrated in studies in which a callosal grid system was used.[17]

\section{Hand Motor Localization}

Hand motor activation can be localized by first identifying the superior and middle frontal gyrus and their abutment at a perpendicular angle to the precentral gyrus. A posterior-pointing genu of the central sulcus, Broca's middle bend, is found between the superior and middle frontal gyri. The PPFM lies in the sulcus and protrudes from the posterior wall of the precentral gyrus at the apex of Broca's middle bend. Hand motor activation is found in this bulge from the precentral gyrus. The superior frontal sulcus, when 
followed to its posterior termination, invariably ends at the middle bend of the central sulcus and is a reliable landmark for the hand motor area.

All patients studied here were found to have hand motor activation in the middle bend of the central sulcus and more specifically in the PPFM. The only other anatomical method of hand motor identification found in the literature takes advantage of fortuitous slices through Broca's pli de passage moyen on the 2D MR image to identify the characteristic knob or omega arising from the posterior wall of the precentral gyrus.[35] Three-dimensional reconstruction of the brain surface has become common in the neurosurgical operating theater as an integral part of MR-based frameless stereotaxy. The advantage of the 3D image is to display the brain more in keeping with the surgeon's view of the cortex. Additionally, the sulcal and gyral pattern is better visualized on the 3D image than the patient's cortex because the leptomeninges and pial vessels are not included in the 3D reconstruction. The method of hand motor identification described here is, therefore, based primarily on the 3D computer rendering of the brain surface, but with careful attention to detail, the middle bend can be identified on the exposed cortex (Fig. 6).

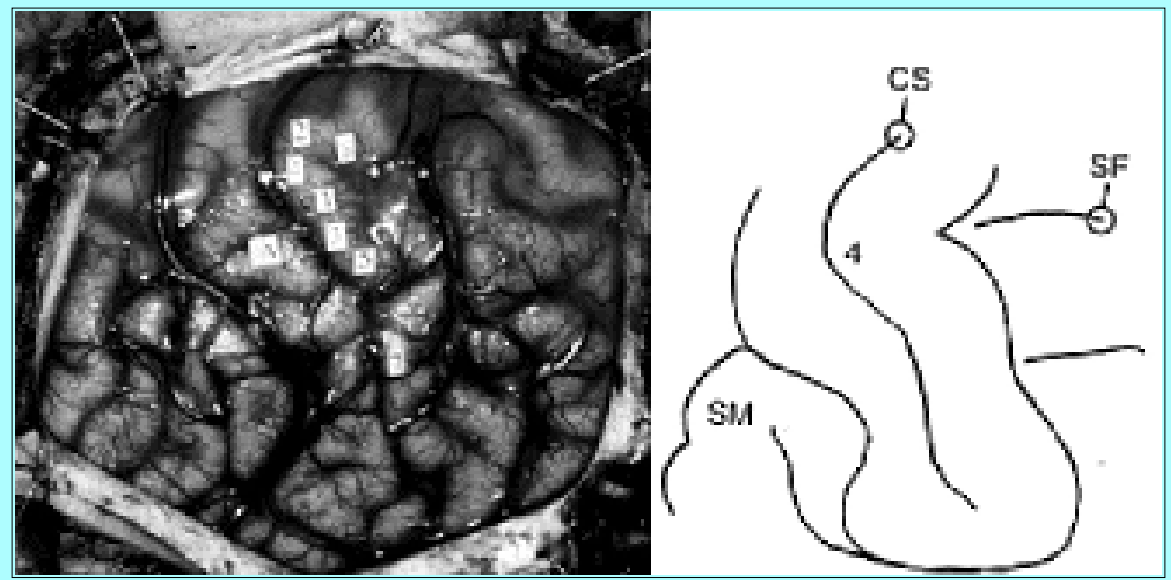

Fig. 6. Left: Intraoperative photograph showing draining veins and pial vessels that tend to obscure the sulcal and gyral anatomy. However, many features can still be distinguished, especially in the perisylvian and central areas. This figure shows a typical craniotomy exposing the central area. Numbers correspond to the sites of cortical stimulation. Tickets $1-5,7$, and 8 are positioned over the precentral gyrus and mark areas in which motor responses were elicited. Stimulation at Ticket 4 produced flexion of all the fingers on the left hand. Right: Tracing of the operative photograph (left) demonstrating the central anatomy. Ticket 4, which represents finger flexion, is seen in Broca's middle bend at the posterior termination of the superior frontal sulcus $(\mathrm{SF})$. $\mathrm{CS}=$ central sulcus; $\mathrm{SM}=$ supramarginal gyrus.

\section{CONCLUSIONS}

Hand motor activation was found at the level of Broca's middle bend in all of the patients studied here, and more precisely, within the PPFM. The hand motor area lies between the superior and middle frontal gyri, on the posterior bank of the precentral gyrus and at the apex of the posterior-pointing middle bend of the central sulcus. Cortical stimulation correlates with the sites of activation seen on functional neuroimaging.[16,20,22,30,36] The findings here are useful, therefore, for localization of hand motor function, and they can be used to guide and plan surgery in or near the central area. The anatomical method of identifying the hand motor area outlined here adds another tool for the clinician to use in 
tackling the problem of localizing motor function.

In this study we demonstrate that constant anatomical relationships in the central area are useful for localization of hand motor function. Additionally, a relationship exists between the functional physiology and the convolutional anatomy of the central area, lending more importance to what previously may have seemed to be a random gyral pattern.

\section{References}

1. Bailey P, von Bonin G: The Isocortex of Man. Urbana, Ill: University of Illinois Press, 1951, pp $61-80$

2. Broca P: Mémoires Sur le Cerveau de l'Homme et des Primates. Paris: Reinwald, 1888, pp 739-804

3. Campbell A: Histological Studies on the Localization of Cerebral Function. Cambridge: University Press, 1905, pp 20-23

4. Catalan MJ, Ishii K, Honda M, et al: Finger somatotopy in human primary somatosensory cortex: a PET study. Neuroimage 7 (Suppl 1):S409, 1998 (Abstract)

5. Collins DL, Neelin P, Peters TM, et al: Automatic 3D intersubject registration of MR volumetric data in standardized Talairach space. J Comput Assist Tomogr 18:192-205, 1994

6. Cunningham DJ: Contributions to the Surface Anatomy of the Cerebral Hemispheres. Cunningham Memoirs No. 7. Dublin: Academy House, 1892, pp 20-24

7. Cushing H: Surgery of the head, in Keen WW (ed): Surgery. Philadelphia: WB Saunders, 1916, pp $17-276$

8. Dejerine J: Anatomie des Centres Nerveux. Paris: Rueff, 1895, Vol 1, pp 248-250

9. Evans AC, Marrett S, Neelin P, et al: Anatomical mapping of functional activation in stereotactic coordinate space. Neuroimage 1:45-53, 1992

10. Evans AC, Marrett S, Torrescorzo J, et al: MRI-PET correlation in three dimensions using a volume-of-interest (VOI) atlas. J Cereb Blood Flow Metab 11:A69-A78, 1991

11. Grafton ST, Woods RP, Mazziotta JC: Within-arm somatotopy in human motor areas determined by positron emission tomography imaging of cerebral blood flow. Exp Brain Res 95:172-176, 1993

12. Jack CR Jr, Thompson RM, Butts RK, et al: Sensory motor cortex: correlation of presurgical mapping with functional MR imaging and invasive cortical mapping. Radiology 190:85-92, 1994

13. Kurth R, Villringer K, Mackert BM, et al: fMRI assessment of somatotopy in human Brodman area 3b by electrical finger stimulation. Neuroreport 9:207-212, 1998

14. Lorensen WE, Cline HE: Marching cubes: a high resolution 3D surface construction algorithm. Comput Graphics 21:163-169, 1987

15. McGonigle DJ, Aston P, Josephs O, et al: Somatotopy of vibrotactile stimulation in SI: an fMRI study. Neuroimage 7 (Suppl):S405, 1998 (Abstract) 
16. Mueller WM, Yetkin FZ, Hammeke TA, et al: Functional magnetic resonance imaging mapping of the motor cortex in patients with cerebral tumors. Neurosurgery 39:515-521, 1996

17. Olivier A, Awad IA: Extratemporal resections, in Engel JJ Jr (ed): Surgical Treatment of the Epilepsies. New York: Raven Press, 1993, pp 489-500

18. Penfield W, Boldrey E: Somatic motor and sensory representation in the cerebral cortex of man as studied by electrical stimulation. Brain 60:389-444, 1937

19. Penfield W, Rasmussen T: The Cerebral Cortex of Man. A Clinical Study of Localization of Function. New York: Macmillan, 1950, pp 429-431

20. Puce A: Comparative assessment of sensorimotor function using functional magnetic resonance imaging and electrophysiological methods. J Clin Neurophysiol 12:450-459, 1995

21. Puce A, Constable RT, Luby ML, et al: Functional magnetic resonance imaging of sensory and motor cortex: comparison with electrophysiological localization. J Neurosurg 83:262-270, 1995

22. Pujol J, Conesa G, Deus J, et al: Presurgical identification of the primary sensorimotor cortex by functional magnetic resonance imaging. J Neurosurg 84:7-13, 1996

23. Rao SM, Binder JR, Hammeke TA, et al: Somatotopic mapping of the human primary motor cortex with functional magnetic resonance imaging. Neurology 45:919-924, 1995

24. Rumeau C, Tzourio N, Murayama N, et al: Location of hand function in the sensorimotor cortex: MR and functional correlation. AJNR 15:567-572, 1994

25. Schiller F: Paul Broca, Explorer of the Brain. New York: Oxford University Press, 1992, pp $165-211$

26. Suk J, Ribary U, Cappell J, et al: Anatomical localization revealed by MEG recordings of the human somatosensory system. Electroencephalogr Clin Neurophysiol 78:185-196, 1991

27. Talairach J, Tournoux P: Co-Planar Stereotaxic Atlas of the Human Brain: 3-Dimensional Proportional System. An Approach to Cerebral Imaging. Rayport M (trans). New York: Thieme, 1988

28. Testut L, Latarjet A: Traité d'Anatomie Humaine. Paris: Doin, 1929, Vol 2, pp 918-938

29. Vinas FC, Zamorano L, Meuller RA, et al: [150]-water PET and intraoperative brain mapping: a comparison in the localization of eloquent cortex. Neurol Res 19:601-608, 1997

30. von Bonin G: Architecture of the precentral motor cortex and some adjacent areas, in Bucy PC (ed): The Precentral Motor Cortex. Urbana, Ill: University of Illinois Press, 1944, pp 32-63

31. von Gelderen P, Ramsey NF, Liu G, et al: Three-dimensional functional magnetic resonance imaging of human brain on a clinical 1.5-T scanner. Proc Natl Acad Sci USA 92:6906-6910, 1995

32. White LE, Andrews TJ, Hulette C, et al: Structure of the human sensorimotor system. I: Morphology and cytoarchitecture of the central sulcus. Cereb Cortex 7:18-30, 1997

33. Wood CC, Spencer DD, Allison T, et al: Localization of human sensorimotor cortex during surgery 
by cortical surface recording of somatosensory evoked potentials. J Neurosurg 68:99-111, 1988

34. Worsley KJ, Evans AC, Marrett S, et al: A three-dimensional statistical analysis for CBF activation studies in human brain. J Cereb Blood Flow Metab 12:900-918, 1992

35. Yousry TA, Schmid UD, Alkadhi H, et al: Localization of the motor hand area to a knob on the precentral gyrus. A new landmark. Brain 120:141-157, 1997

36. Yousry TA, Schmid UD, Jassoy AG, et al: Topography of the cortical motor hand area: prospective study with functional MR imaging and direct motor mapping at surgery. Radiology 195:23-29, 1995

Manuscript received March 25, 1999.

Accepted in final form July 19, 1999.

Address reprint requests to: Warren Boling, M.D., Department of Neurosurgery, Montreal Neurological Institute and Hospital, 3801 rue University, Montreal, Quebec H3A-2B4, Canada. email: warren@bic.mni.mcgill.ca.

\section{Appendix}

The method of identifying hand motor activation by using brain surface landmarks was presented to observers as follows: the region of PET activation is identified reliably on the surface of the brain by its anatomical relationship to nearby structures. After identification of the central sulcus, the superior and middle frontal gyrus are seen to abut the precentral gyrus at a right angle. The superior and middle frontal gyrus also are usually continuous with, and arise from, the precentral gyrus. A bend or genu pointing posteriorly in the precentral gyrus is seen constantly between the superior and the middle frontal gyrus. The apex of this posteriorly pointing genu is the location of hand motor activation seen on PET activation studies. Also, the precentral gyrus is usually wider at the point of hand motor activation, thereby providing an additional aid to identification. 